PROCEEDINGS OF THE

AMERICAN MATHEMATICAL SOCIETY

Volume 117, Number 1, January 1993

\title{
MAPPING SPACES OF COMPACT LIE GROUPS AND $p$-ADIC COMPLETION
}

\author{
DAVID BLANC AND DIETRICH NOTBOHM
}

(Communicated by Frederick R. Cohen)

\begin{abstract}
If BG. BH are the classifying spaces of compact Lie groups. with $\mathbf{H}$ connected, then the mapping space functor $\operatorname{map}(\mathbf{B G},-)$ commutes with $p$-completion on $\mathbf{B H}:$ i.e. for each $f: \mathbf{B G} \rightarrow \mathbf{B H}$ the component $\left(\operatorname{map}(\mathbf{B G}, \mathbf{B H})_{f}\right)_{p}^{\wedge}$ is $p$-complete, and is homotopy equivalent to $\operatorname{map}\left(\mathbf{B G}, \mathbf{B H}_{p}^{\wedge}\right)_{i \circ f}$.
\end{abstract}

\section{INTRODUCTION}

In studying $\operatorname{map}(\mathbf{B G}, \mathbf{B H})$, the space of maps between the classifying spaces of two compact Lie groups, it is often useful to know whether the $p$-adic completion commutes with the functor $\operatorname{map}(\mathbf{B G},--)$; special cases where this occurs were used, for example, in [DZ, JMO, N2, NS]. Here we present a more general result in this direction:

1.1. Theorem. Let $\mathbf{G}$ and $\mathbf{H}$ be compact Lie groups, with $\mathbf{H}$ connected; let $p$ be a prime, and $i: \mathbf{B H} \rightarrow \mathbf{B H} \wedge$ the natural inclusion. Then for any map $f: \mathbf{B G} \rightarrow \mathbf{B H}$, the corresponding component of the mapping space, $\operatorname{map}\left(\mathbf{B G}, \mathbf{B H}_{p}^{\wedge}\right)_{i \circ f}$, is p-complete, and

$$
\left(\operatorname{map}(\mathbf{B G}, \mathbf{B H})_{f}\right)_{p}^{\wedge} \stackrel{\simeq}{\leftrightarrows} \operatorname{map}\left(\mathbf{B G}, \mathbf{B H}{ }_{p}^{\wedge}\right)_{i \circ f}
$$

is a homotopy equivalence.

The $p$-adic completion of a space $\mathbf{X}$ that we refer to is the $\left(\mathbb{F}_{p}\right)_{\infty} \mathbf{X}$ of [BK, I, §4.2], which we denote by $\mathbf{X}_{p}^{\wedge}$. However, unless $\mathbf{X}$ is nilpotent (e.g., simply-connected), $\quad \mathbf{X}_{p}^{\wedge}$ need not be $p$-complete in the sense of [BK, I, $\S 5 \&$ VII, §2], and so it enjoys few of the properties associated with completion. In particular, unless $\mathbf{X}_{p}^{\wedge}$ is $p$-complete, the natural map $i: \mathbf{X} \rightarrow \mathbf{X}_{p}^{\wedge}$ will not induce an isomorphism in $\mathbb{F}_{p}$-homology, so $\mathbf{X}_{p}^{\wedge}$ will not be the $H_{\star}\left(-; \mathbb{F}_{p}\right)$-localization of $\mathbf{X}$ (cf. [BK, $\S 2.1])$ and $\left(\mathbf{X}_{p}^{\wedge}\right)_{p}^{\wedge} \neq \mathbf{X}_{p}^{\wedge}$.

In $\$ 2$ we list some facts about $\mathbb{Z}^{\wedge}$-modules needed to prove the theorem. In $\& 3$ 
In $\S 4$ the Bousfield-Kan spectral sequence is used to prove $p$-completeness. The required homotopy equivalence is shown in $\S 5$.

\section{Finitely Generated $\mathbb{Z}_{p}^{\wedge}$-MODUles}

Let $\mathscr{F}$ denote the class of finitely generated $\mathbb{Z}_{p}^{\wedge}$-modules, where $\mathbb{Z}_{p}^{\wedge}$ is the ring of $p$-adic integers, and let $\mathscr{F}^{\prime}=\mathscr{F} \cup\{G: G$ is a finite $p$-group $\}$.

2.1. Lemma. If $\mathbf{X}$ is a connected space with $\pi_{k} \mathbf{X} \in \mathscr{F}^{\prime}$ for each $k \geq 1$, then

(1) $H_{\star}\left(\mathbf{X} ; \mathbb{F}_{p}\right)$ is of finite type, that is, $H_{k}\left(\mathbf{X} ; \mathbb{F}_{p}\right)$ is finite for each $k \geq 0$;

(2) $\mathbf{X}$ is p-complete and $\mathbb{F}_{q}$-acyclic for any prime $q \neq p$, that is, $\widetilde{H}_{\star}\left(\mathbf{X} ; \mathbb{F}_{q}\right)$ $=0$.

Proof. Any $M \in \mathscr{F}$ is isomorphic to $N \otimes \mathbb{Z}_{p}^{\wedge}$, where $N$ is a finitely generated abelian group. Thus $\mathbf{K}(M, n) \simeq \mathbf{K}(N, n)_{p}^{\wedge}$, which is $p$-complete (see [BK, VI, 5.2]), and so $H_{\star}\left(\mathbf{K}(M, n) ; \mathbb{F}_{p}\right)$ is of finite type for all $n \geq 1$. Therefore, if $\mathbf{Y}$ is a simply-connected space with each $\pi_{i} \mathbf{Y} \in \mathscr{F}$, by induction on its Postnikov system, we see $H_{\star}\left(\mathbf{Y} ; \mathbb{F}_{p}\right)$ is of finite type.

Now assume $\pi_{1} \mathbf{X}=G \in \mathscr{F}^{\prime}$ and consider the universal covering fibration for $\mathbf{X}$;

$$
\tilde{X} \rightarrow \mathbf{X} \rightarrow \mathbf{K}(G, 1) .
$$

The action of $G$ on the universal covering space $\widetilde{\mathbf{X}}$ makes $H_{t}\left(\tilde{\mathbf{X}} ; \mathbb{F}_{p}\right)$ into a $G$-module, and one has a Leray-Cartan spectral sequence (cf. [CE, XVI, §9]), with

$$
E_{s, t}^{2} \cong H_{s}\left(G ; H_{t}\left(\widetilde{\mathbf{X}} ; \mathbb{F}_{p}\right)\right) \Rightarrow H_{t+s}\left(\mathbf{X} ; \mathbb{F}_{p}\right) .
$$

Now for fixed $t$, let $V=H_{t}\left(\tilde{\mathbf{X}} ; \mathbb{F}_{p}\right)$ and let $\phi: G \rightarrow \operatorname{Aut}(V)$ describe the $\pi_{1}$-action. $\operatorname{Aut}(V)$ is finite, and if $G \in \mathscr{F}$ then $G$ is $q$-divisible for any $q$ prime to $p$, so in any case $\operatorname{Im}(\phi) \subseteq \operatorname{Aut}(V)$ is a finite $p$-group. Thus $G$ acts nilpotently on $V$ (cf. [BK, II, 5.2]): that is, there is a filtration $0=V_{0} \subset V_{1} \subset$ $\cdots V_{i} \cdots \subset V_{n}=V$ of $G$-modules such that $G$ acts trivially on each $V_{i} / V_{i-1}$.

Using the short exact sequences $0 \rightarrow V_{i-1} \rightarrow V_{i} \rightarrow V_{i} / V_{i-1} \rightarrow 0$, we see by induction on $i$ that each $H_{s}\left(G ; V_{i}\right)$-and so in particular $H_{s}(G ; V) \cong E_{s, t}^{2}$-is finite. Thus $H_{\star}\left(\mathbf{X} ; \mathbb{F}_{p}\right)$ is of finite type.

Furthermore, because $G$ acts nilpotently on $V$, by the mod- $\mathbb{F}_{p}$ fiber lemma of [BK,II, 5.1] the universal covering $(\star)$ remains a fibration after $p$-completion: $(\star)_{p}^{\wedge}$

$$
\tilde{\mathbf{X}}_{p}^{\wedge} \rightarrow \mathbf{X}_{p}^{\wedge} \rightarrow \mathbf{K}(G, 1)_{p}^{\wedge} .
$$

Since $G=\pi_{1} \mathbf{X} \in \mathscr{F}^{\prime}, \mathbf{K}(G, 1)$ is $p$-complete; similarly $\widetilde{\mathbf{X}} \simeq \widetilde{\mathbf{X}}_{p}^{\wedge}$ (being nilpotent, with $\pi_{k} \tilde{\mathbf{X}} \in \mathscr{F}$ ). The Five Lemma, applied to the natural map from the long exact sequence of $(\star)$ to that of $(\star)_{p}^{\wedge}$, shows $\pi_{\star} \mathbf{X} \rightarrow \pi_{\star}\left(\mathbf{X}_{p}^{\wedge}\right)$ is an isomorphism, so $\mathbf{X}$ is $p$-complete. Since $\widetilde{H}_{\star} \widetilde{\mathbf{X}}=0=\widetilde{H}_{\star} \mathbf{K}(G, 1)$ for $q \neq p$ by [BK, VI, 5.6], the same holds for $\mathbf{X}$.

2.2. Corollary. Let $\mathbf{X}$ be a pointed connected space such that $\pi_{k} \mathbf{X} \in \mathscr{F}$ for $k \geq 2$, and suppose that $\pi_{1} \mathbf{X}$ has a finite normal series

$$
1=G_{0} \triangleleft G_{1} \triangleleft \cdots \triangleleft G_{n-1} \triangleleft G_{n}=\pi_{1} \mathbf{X},
$$

where each $G_{i} / G_{i-1} \in \mathscr{F}^{\prime}$. Then $\mathbf{X}$ is p-complete and $\mathbb{F}_{q}$-acyclic for $q \neq p$. 
Proof. For each $i=1, \ldots, n$, let $\quad \mathbf{X}_{i-1} \rightarrow \mathbf{X}_{i} \rightarrow \mathbf{K}\left(G_{i} / G_{i-1}, 1\right)$ be the covering fibration corresponding to the short exact sequence $1 \rightarrow G_{i-1} \rightarrow G_{i} \rightarrow$ $G_{i} / G_{i-1} \rightarrow 1$ (where $\mathbf{X}_{0}=\widetilde{\mathbf{X}}$ and $\mathbf{X}_{n}=\mathbf{X}$ ). As above, $\mathbf{K}\left(G_{i} / G_{i-1}, 1\right)$, and by induction also $\mathbf{X}_{i-1}$, are $p$-complete and $\mathbb{F}_{q}$-acyclic, with $\mathbb{F}_{p}$-homology of finite type. The same then holds for $\mathbf{X}_{i}$, too, by the covering-space argument in the proof of Lemma 2.1, and thus for $\mathbf{X}$.

\subsection{Lemma. For $A, C \in \mathscr{F}$ :}

(1) If $0 \rightarrow A \rightarrow B \rightarrow C \rightarrow 0$ is a short exact sequence of abelian groups, then $B \in \mathscr{F}$.

(2) Any group homomorphism $f: C \rightarrow A$ is $\mathbb{Z}_{p}^{\wedge}$-linear.

Proof. It is enough to show that the forgetful functor induces isomorphisms

$$
\operatorname{Ext}_{\mathbb{Z}_{p}^{\wedge}}^{1}(C, A) \cong \operatorname{Ext}_{\mathbb{Z}}^{1}(C, A) \text { and } \operatorname{Hom}_{\mathbb{Z}_{p}^{\wedge}}(C, A) \cong \operatorname{Hom}_{\mathbb{Z}}(C, A)
$$

As above, write $A \cong A^{\prime} \otimes \mathbb{Z}_{p}^{\wedge}, C \cong C^{\prime} \otimes \mathbb{Z}_{p}^{\wedge}$, for finitely generated abelian groups $A^{\prime}, C^{\prime}$. Since Ext and Hom commute with finite direct sums, it is enough to consider cyclic $C$ and $A$, that is, each either $\mathbb{Z}_{p}^{\wedge}$ or $\mathbb{Z} / p^{r}$ for some $r$.

By the Change of Rings Theorem (see [HS, IV, Theorem 12.2]) we know

$$
\operatorname{Ext}_{\mathbb{Z}_{p}^{\wedge}}^{n}(C, A) \cong \operatorname{Ext}_{\mathbb{Z}_{p}^{\wedge}}^{n}\left(C^{\prime} \otimes \mathbb{Z}_{p}^{\wedge}, A\right) \stackrel{\cong}{\rightarrow} \operatorname{Ext}_{\mathbb{Z}}^{n}\left(C^{\prime}, A\right) \quad(n \geq 0),
$$

so (1) is satisfied when $C$ is torsion and thus $C=C^{\prime}$.

Now let $C=\mathbb{Z}_{p}^{\wedge}$.

(1) If $A=\mathbb{Z}_{p}^{\wedge}$ then $\operatorname{Ext}_{\mathbb{Z}}^{1}(C, A)=0$ by [Ha, Proposition 2.1].

(2) If $A=\mathbb{Z} / p^{r}$, tensor $0 \rightarrow \mathbb{Z} \rightarrow \mathbb{Q} \rightarrow \mathbb{Q} / \mathbb{Z} \rightarrow 0$ with $\mathbb{Z}_{p}^{\wedge}$ to get the exact sequence $0=\operatorname{Tor}\left(\mathbb{Q} / \mathbb{Z}, \mathbb{Z}_{p}^{\wedge}\right) \rightarrow \mathbb{Z}_{p}^{\wedge} \rightarrow \mathbb{Q} \otimes \mathbb{Z}_{p}^{\wedge} \rightarrow(\mathbb{Q} / \mathbb{Z}) \otimes \mathbb{Z}_{p}^{\wedge} \rightarrow 0$. Applying $\operatorname{Ext}_{\mathbb{Z}}^{1}(-, A)$ to this, we see that $\operatorname{Ext}_{\mathbb{Z}}^{1}\left(\mathbb{Z}_{p}^{\wedge}, \mathbb{Z} / p^{r}\right)=0$ since $\mathbb{Z}_{p}^{\wedge} \otimes \mathbb{Q}$ is a $\mathbb{Q}$-vector space and $\operatorname{Ext}_{\mathbb{Z}}^{1}\left(\mathbb{Q}, \mathbb{Z} / p^{r}\right)=0$.

We clearly also have $\operatorname{Ext}_{\mathbb{Z}_{\hat{p}}^{\wedge}}^{1}\left(\mathbb{Z}_{p}^{\wedge}, A\right)=0$ for any $A$.

Finally, $\operatorname{Hom}_{\mathbb{Z}_{p}^{\wedge}}\left(\mathbb{Z}_{p}^{\wedge}, A\right) \cong A$ for any $A \in \mathscr{F}$ while $\operatorname{Hom}_{\mathbb{Z}}\left(\mathbb{Z}_{p}^{\wedge}, \mathbb{Z} / p^{r}\right) \cong$ $\mathbb{Z} / p^{r}$, so

$$
\begin{aligned}
& \operatorname{Hom}_{\mathbb{Z}}\left(\mathbb{Z}_{p}^{\wedge}, \mathbb{Z}_{p}^{\wedge}\right)=\operatorname{Hom}_{\mathbb{Z}}\left(\mathbb{Z}_{p}^{\wedge}, \underset{\lim }{\longleftarrow} \mathbb{Z} / p^{r}\right) \\
& \cong \lim \operatorname{Hom}_{\mathbb{Z}}\left(\mathbb{Z}_{p}^{\wedge}, \mathbb{Z} / p^{r}\right) \cong \lim \mathbb{Z} / p^{r} \cong \mathbb{Z}_{p}^{\wedge} .
\end{aligned}
$$

Thus $\operatorname{Hom}_{\mathbb{Z}}\left(\mathbb{Z}_{p}^{\wedge}, A\right) \cong A$ for any $A \in \mathscr{F}$, too. The required isomorphism is readily verified.

\section{THE MOD- $p$ APPROXIMATION OF BG}

In order to prove Theorem 1.1 for more general $\mathbf{G}$, we start with the known case when $\mathbf{G}$ is $p$-toral, i.e., $\pi_{0} \mathbf{G}$ is a finite $p$-group and the identity component of $\mathbf{G}$ is a torus. Then we have 
3.1. Lemma. If $\mathbf{P}$ is a p-toral group and $\mathbf{H}$ is a connected compact Lie group, then for any $\left.f: \mathbf{B P} \rightarrow \mathbf{B H},\left(\operatorname{map}(\mathbf{B P}, \mathbf{B H})_{f}\right)_{p}^{\wedge} \rightarrow \operatorname{map}(\mathbf{B P}, \mathbf{B H})_{p}^{\wedge}\right)_{i \circ f}$ is a homotopy equivalence.

This is contained in [JMO, Theorem 3.2]; we give an outline of the proof:

By [N1, Theorem 1.1], $f \simeq B \rho$ for some homomorphism $\rho: \mathbf{P} \rightarrow \mathbf{H}$; let $\mathbf{C}(\rho)$ denote its centralizer. The homomorphism $\mathbf{C}(\rho) \times \mathbf{P} \rightarrow \mathbf{H}$ passes to classifying spaces and has an adjoint $\mathbf{B C}(\rho) \rightarrow \operatorname{map}(\mathbf{B P}, \mathbf{B H})_{B \rho}$, or if we first complete,

$$
\mathbf{B C}(\rho)_{p}^{\wedge} \rightarrow \operatorname{map}\left(\mathbf{B P}, \mathbf{B H}_{p}^{\wedge}\right)_{i \circ B \rho} .
$$

The first map induces an $H_{\star}\left(-; \mathbb{F}_{p}\right)$-isomorphism by [N1], and so a homotopy equivalence after completion (see [BK, I, 5.5]), while the second is shown in [JMO, loc. cit.] to be a homotopy equivalence.

3.2. Remark. Since $\mathbf{C}(\rho)$ is compact and $\pi_{0} \mathbf{C}(\rho)$ is a finite $p$-group (cf. [JMO, Proposition A.4]), the homotopy groups $\pi_{k}\left(\operatorname{map}\left(\mathbf{B P}, \mathbf{B H}_{p}^{\wedge}\right)_{i \circ B \rho}\right)$ are finitely generated $\mathbb{Z}_{p}^{\wedge}$-modules for $k \geq 2$ and a finite $p$-group for $k=1$.

We now recall some results of Jackowski, McClure, and Oliver on the mod- $p$ approximation of $\mathbf{B G}$ :

For any compact Lie group $\mathbf{G}$, let $\mathscr{O}_{p}(\mathbf{G})$ denote the full subcategory of the orbit category $\mathscr{O}(\mathbf{G})$ whose objects are homogenous spaces $\mathbf{G} / \mathbf{P}$ where $\mathbf{P}$ is a $p$-toral group and whose morphisms are G-maps. In [JMO, 1.3], Jackowski, McClure, and Oliver define a full subcategory $\mathscr{R}_{p}(\mathbf{G}) \subset \mathscr{O}_{p}(\mathbf{G})$ (containing $\mathbf{G} / \mathbf{P}$ only for certain “ $p$-stubborn" $\mathbf{P}$ 's), which has the property that

$$
\underset{\mathscr{R}_{p}(\mathbf{G})}{\operatorname{holim}} \mathbf{E G} \times \mathbf{G}(\mathbf{G} / \mathbf{P}) \rightarrow \mathbf{B G}
$$

is a $H_{\star}\left(-; \mathbb{F}_{p}\right)$-isomorphism. Here holim denotes the homotopy direct limit of [BK, XII, §2], and $\mathbf{E G} \times \times_{\mathbf{G}}(\mathbf{G} / \mathbf{P}) \cong \mathbf{E G} / \mathbf{P} \simeq \mathbf{B P}$.

Recall from [BK, I, $\S 4]$ that for any space $\mathbf{X}$, the $p$-completion is obtained as the total space (i.e., homotopy inverse limit) of a certain cosimplicial space: $\mathbf{X}_{p}^{\wedge} \stackrel{\text { def }}{=} \operatorname{Tot}(\underset{\sim}{\mathbb{F}} \mathbf{X})^{\bullet}$, where each space $(\underset{\sim}{\mathbb{F}} \mathbf{X})^{k}$ is homotopy equivalent to an $\mathbb{F}_{p^{-}}$ GEM, i.e., a product of $\mathbf{K}\left(\mathbb{F}_{p}, n_{i}\right)$ 's. Therefore, for any space $\mathbf{Z}$, we have

$$
\left.\operatorname{map}\left(\mathbf{Z}, \mathbf{X}_{p}^{\wedge}\right)=\operatorname{map}\left(\mathbf{Z}, \operatorname{Tot}\left(\underset{\sim}{\mathbb{F}_{p}} \mathbf{X}\right)^{\bullet}\right) \cong \operatorname{Tot}\left(\operatorname{map}\left(\mathbf{Z}, \underset{\sim}{\mathbb{F}_{p}} \mathbf{X}\right)^{\bullet}\right)\right)
$$

(see [BK, XI, 4.4, 7.6]), so the space of maps into a $p$-completion is the total space of a cosimplicial $\mathbb{F}_{p}$-GEM, too.

Now if $f: \mathbf{Y} \rightarrow \mathbf{Z}$ is an $H_{\star}\left(-; \mathbb{F}_{p}\right)$-isomorphism, it induces a homotopy equivalence $\operatorname{map}\left(\mathbf{Z}, \mathbf{K}\left(\mathbb{F}_{p}, n\right)\right) \stackrel{f^{*}}{\rightarrow} \operatorname{map}\left(\mathbf{Y}, \mathbf{K}\left(\mathbb{F}_{p}, n\right)\right)$, and so $\left.\operatorname{map}(\mathbf{Z}, \underset{\sim}{\underset{\sim}{\mathbb{F}} \mathbf{X}})^{k}\right)$ $\stackrel{f^{*}}{\rightarrow} \operatorname{map}\left(\mathbf{Y},(\underset{\sim}{\mathbb{F}} \mathbf{X})^{k}\right)$ is a homotopy equivalence for each $k \geq 0$. Therefore, by [BK, XI, 5.6] the same is true for the Tot's, and thus $\operatorname{map}\left(\mathbf{Z}, \mathbf{X}_{p}^{\wedge}\right) \stackrel{f^{*}}{\rightarrow} \operatorname{map}\left(\mathbf{Y}, \mathbf{X}_{p}^{\wedge}\right)$ is a homotopy equivalence. Since

$$
\operatorname{map}\left(\stackrel{\text { holim }}{\longrightarrow} \mathbf{Y}_{i}, \mathbf{X}\right)=\underline{\text { holim }} \operatorname{map}\left(\mathbf{Y}_{i}, \mathbf{X}\right)
$$


for any diagram $\left\{\mathbf{Y}_{i}\right\}$ (cf. [BK, XII, 4.1]), we have a natural homotopy equivalence

$$
\operatorname{map}\left(\mathbf{B G}, \mathbf{B H}_{p}^{\wedge}\right) \rightarrow \underset{\mathscr{R}_{p}(G)}{\operatorname{holim}} \operatorname{map}\left(\mathbf{E G} / \mathbf{P}, \mathbf{B H}_{p}^{\wedge}\right) \text {. }
$$

Thus, if we restrict a map $f: \mathbf{B G} \rightarrow \mathbf{B H}$ to $\mathbf{B P} \hookrightarrow \mathbf{B G}$ (for some $\mathbf{G} / \mathbf{P}$ in $\left.\mathscr{R}_{p}(\mathbf{G})\right)$, we see that

$$
\operatorname{map}\left(\mathbf{B G}, \mathbf{B H} H_{p}^{\wedge}\right)_{i \circ f} \rightarrow \underbrace{\operatorname{holim}}_{\mathscr{R}_{p}(G)} \operatorname{map}\left(\mathbf{E G} / \mathbf{P}, \mathbf{B} \mathbf{H}_{p}^{\wedge}\right)_{\left.i \circ f\right|_{\mathbf{B P}}}
$$

is the inclusion of a component (the homotopy inverse limit need not be connected!).

\section{COSIMPLICAL SPACES}

Let sk $\mathscr{R}_{p}(\mathbf{G})$ be a skeleton of $\mathscr{R}_{p}(\mathbf{G})$, that is, a full subcategory of $\mathscr{R}_{p}(\mathbf{G})$, containing a single representative of each isomorphism type of its objects. This is a finite category, since $\mathscr{R}_{p}(\mathbf{G})$ has finitely many isomorphism types of objects, and finitely many morphisms between them (cf. [JMO, Proposition 1.6]).

Given a map $f: \mathbf{B G} \rightarrow \mathbf{B H}$ as above, consider the finite diagram of spaces

$$
\underline{\mathbf{X}}=\left\{\mathbf{X}_{\mathbf{P}}\right\}_{\mathbf{G} / \mathbf{P} \in \mathbf{s k} \mathscr{R}_{p}(\mathbf{G})}, \quad \text { where } \mathbf{X}_{\mathbf{P}}=\operatorname{map}\left(\mathbf{B P}, \mathbf{B} \mathbf{H}_{p}^{\wedge}\right)_{\left.i \circ f\right|_{\mathbf{B P}}} .
$$

By cosimplicial replacement (see [BK, XI, §5]) we obtain a cosimplicial space $\mathbf{Y}^{\bullet}$, with

$$
\mathbf{Y}^{n}=\prod_{\mathbf{G} / \mathbf{P}_{i_{0}} \rightarrow \cdots \rightarrow \mathbf{G} / \mathbf{P}_{i_{n}}} \mathbf{X}_{\mathbf{P}_{i_{0}}}
$$

(where the product, over all possible sequences of $n$ composable morphisms in sk $\mathscr{R}_{p}(\mathbf{G})$, is finite), such that $\operatorname{holim}_{\mathrm{sk}} \mathscr{R}_{p}(\mathbf{G})\left\{\mathbf{X}_{\mathbf{P}}\right\} \cong \operatorname{Tot} \mathbf{Y}^{\bullet}$.

Now if $\mathbf{Z}^{\bullet}$ is the cosimplicial replacement of the analogous infinite diagram of $\mathbf{X}_{\mathbf{P}}$ 's for the full category $\mathscr{R}_{p}(\mathbf{G})$, then the equivalence of categories sk $\mathscr{R}_{p}(\mathbf{G}) \hookrightarrow \mathscr{R}_{p}(\mathbf{G})$ (with noncanonical inverse $\mathscr{R}_{p}(\mathbf{G}) \rightarrow$ sk $\mathscr{R}_{p}(\mathbf{G})$ ) induces a homotopy equivalence $\operatorname{Tot} \mathbf{Y}^{\bullet} \cong$ $\cong$ Tot $\mathbf{Z}^{\bullet}$, so that up to homotopy the natural map of (2) above is the inclusion of one component in $\operatorname{Tot} \mathbf{Y}^{\bullet}$ :

$$
\operatorname{map}\left(\mathbf{B G}, \mathbf{B} \mathbf{H}_{p}^{\wedge}\right)_{i \circ f} \hookrightarrow \underbrace{\operatorname{holim}}_{\mathscr{R}_{p}(G)}\left\{\mathbf{X}_{\mathbf{P}}\right\} \simeq \operatorname{Tot} \mathbf{Y}^{\bullet} .
$$

We choose a basepoint $y_{0} \in \operatorname{Tot} \mathbf{Y}^{\bullet}$ corresponding to the map $i \circ f$.

4.1. Lemma. For any $f: \mathbf{B G} \rightarrow \mathbf{B H}$, the space $\operatorname{map}\left(\mathbf{B G}, \mathbf{B} \mathbf{H}_{p}^{\wedge}\right)_{i \circ f}$ is $p$ complete and $\mathbb{F}_{q}$-acyclic for $q \neq p$.

Proof. Consider the Bousfield-Kan spectral sequence for $\mathbf{Y}^{\bullet}$ as above (more precisely, for the component of $y_{0}$ in $\operatorname{Tot}^{\bullet}\left(\mathrm{cf}\right.$. [B2, §2])) with $E_{2}^{s, t} \cong$ $\pi^{s} \pi_{t} \mathbf{Y}^{\bullet}$.

For $t \geq 2$, the construction of $\mathbf{Y}^{\bullet}$ and Remark 3.2 imply that $\pi_{t} \mathbf{Y}^{s} \in \mathscr{F}$ and all the cosimplicial morphisms of $\pi_{t} \mathbf{Y}^{\bullet}$ are $\mathbb{Z}_{p}^{\wedge}$-linear by Lemma $2.3(\mathrm{~b})$; hence $E_{2}^{s, t} \in \mathscr{F}$. For $t=1, E_{2}^{0,1}$ is a subgroup of $\pi_{1} \mathbf{Y}^{0} \cong \prod \pi_{1} \mathbf{X}_{\mathbf{P}}$, and so is itself a finite $p$-group by Remark 3.2.

Moreover, if $t \geq 2$, the differentials $d_{r}: E_{r}^{s, t} \rightarrow E_{r}^{s+r, t+r-1}$ are homomorphisms, and thus $\mathbb{Z}_{p}^{\wedge}$-linear, for $t>s \geq 0$. Therefore, $E_{r}^{s, t} \in \mathscr{F}$ for $r \leq \infty$, 
if $t>s \geq 0$ or $t=s \geq r$. For $t=1$ we have $E_{r}^{0,1} \subseteq E_{r-1}^{0,1} \subseteq E_{2}^{0,1} \quad$ (cf. [B2, $\S 2.4])$, so $E_{r}^{0,1}$ is a finite $p$-group.

Since $E_{2}^{s, t} \cong \lim _{\mathscr{R}_{p}(G)}{ }^{s} \pi_{t} \underline{\mathbf{X}}$ by [BK, XI, 7.1], Lemma 4.2 below, applied to the functors

$$
\pi_{t}\left(\mathbf{E G} \times_{\mathbf{G}}-\right): \mathscr{R}_{p}(\mathbf{G}) \rightarrow \mathbb{Z}_{p}^{\wedge} \text {-Mod },
$$

shows that there is an $N$ such that $E_{2}^{s, t}=0$ for $s>N$ and $t \geq 2$.

This in turn implies the complete convergence of the spectral sequence (see [B2, §4.5]): thus, for each $t \geq 1$ there is a finite tower of epimorphisms

$$
\pi_{t}\left(\operatorname{Tot} \mathbf{Y}^{\bullet}, y_{0}\right) \cong Q_{N} \pi_{t} \rightarrow \cdots Q_{s} \pi_{t} \rightarrow Q_{s-1} \pi_{t} \rightarrow \cdots Q_{0} \pi_{t} \rightarrow Q_{-1} \pi_{t}=1,
$$

where $Q_{s} \pi_{t}=\operatorname{im}\left\{\pi_{t}\left(\operatorname{Tot} \mathbf{Y}^{\bullet}, y_{0}\right) \rightarrow \pi_{t}\left(\operatorname{Tot}_{s} \mathbf{Y}^{\bullet}, y_{0}\right)\right\} \quad$ (cf. [BK, IX, §5.3]), and for each $s \geq 0$ there is a short exact sequence

$$
1 \rightarrow E_{\infty}^{s, s+t} \rightarrow Q_{s} \pi_{t} \rightarrow Q_{s-1} \pi_{t} \rightarrow 1 .
$$

Now for $t \geq 2$ we have $E_{\infty}^{s, s+t} \in \mathscr{F}$. Therefore, Lemma 2.3(a) implies (by induction on $s)$ that $Q_{s} \pi_{t} \in \mathscr{F}$ for all $s$, and so $\pi_{t}\left(\operatorname{Tot} \mathbf{Y}^{\bullet}, y_{0}\right)$ is in $\mathscr{F}$, too.

For $t=1$ we obtain a finite normal series

$$
0=G_{0} \triangleleft G_{1} \triangleleft \cdots \triangleleft G_{N} \triangleleft G_{N+1}=\pi_{1}\left(\operatorname{Tot} \mathbf{Y}^{\bullet}, y_{0}\right),
$$

where $G_{i} / G_{i-1}=E_{\infty}^{N-i+1, N-i+2}$ is in $\mathscr{F}$ for $1 \leq i \leq N$ and $G_{N+1} / G_{N}=E_{\infty}^{0,1}$ is a finite $p$-group. Thus Corollary 2.2 applies, and the component of $y_{0}$ in Tot $\mathbf{Y}^{\bullet}$ is $p$-complete, and $\mathbb{F}_{q}$-acyclic for $q \neq p$.

The following lemma appeared in an earlier version of [JMO].

4.2. Lemma. If $\mathbf{G}$ is any compact Lie group and $p$ a prime, there is an $N$ such that for any contravariant functor

$$
F: \mathscr{R}_{p}(\mathbf{G}) \rightarrow \mathbb{Z}_{p}^{\wedge} \text {-Mod }
$$

we have $\stackrel{\lim }{\longleftarrow}_{\mathscr{R}_{p}(G)} F=0$ for $s>N$.

Proof. The homotopy direct limit $E \mathscr{R}_{p}(\mathbf{G})=$ holim $_{\mathscr{R}_{p}(G)} \mathbf{G} / \mathbf{P}$ is a $\mathbf{G}$-space, and holim $_{\mathscr{R}_{p}(G)}^{s} F \cong H_{\mathbf{G}}^{s}\left(E \mathscr{R}_{p}(\mathbf{G}) ; F\right)$ for all $s \geq 0$ by [JMO, Theorem 1.7]. Here $\overleftarrow{H_{\mathbf{G}}^{*}(-; F)}$ denotes equivariant cohomology with the functor $F$ as coefficient system (see [I, 2.2]).

By [JMO, Proposition 1.2, Theorem 2.14], there exists a finite dimensional G-complex $\mathbf{X}$ with finitely many orbit types and a $\mathbf{G}-\mathbb{F}_{p}$-isomorphism $f: \mathbf{X} \rightarrow$ $E \mathscr{R}_{p}(\mathbf{G})$; that is, a G-equivariant map $f$ such that $f^{\mathbf{H}}: \mathbf{X}^{\mathbf{H}} \rightarrow\left(E \mathscr{R}_{p}(\mathbf{G})\right)^{\mathbf{H}}$ is an $H_{\star}\left(-; \mathbb{F}_{p}\right)$-isomorphism on $\mathbf{H}$-fixed point sets for any $\mathbf{H} \subseteq \mathbf{G}$.

Since each $H_{k}\left(\left(E \mathscr{R}_{p}(\mathbf{G})\right)^{\mathbf{H}} ; \mathbb{Z}\right)$ is finitely generated (see [JMO, Proposition $1.1]), f^{\mathbf{H}}$ is in fact an isomorphism in $\mathbb{Z}_{p}^{\wedge}$-homology for each $\mathbf{H}$, and therefore $f$ is a $\mathbf{G}-\mathbb{Z}_{p}^{\wedge}$-homology isomorphism; by [JMO, A.13] this implies that $H_{\mathbf{G}}^{\star}\left(E \mathscr{R}_{p}(\mathbf{G}) ; F\right) \cong H_{\mathbf{G}}^{\star}(\mathbf{X} ; F)$ for any $\mathbb{Z}_{p}^{\wedge}$-module valued coefficient system.

Now one can filter $\mathbf{X}$ by $\mathbf{G}$-skeleta $\mathbf{X}_{0} \subset \mathbf{X}_{1} \subset \cdots \subset \mathbf{X}_{i} \subset \cdots \subset \mathbf{X}_{k}=\mathbf{X}$ so that $\mathbf{X}_{i} / \mathbf{X}_{i-1}$ contains a single orbit type $\mathbf{G} / \mathbf{P}_{i}$. If $N$ is the dimension of $\mathbf{X}$, by induction on the $\mathbf{X}_{i}$ one then shows (as in the proof of [JMO, A.13]) that $H_{\mathbf{G}}^{s}(\mathbf{X} ; F)=0$ for $s>N$. 
5. THE HOMOTOPY EQUIVALENCE

For a connected compact Lie group $\mathbf{H}$, consider the arithmetic square

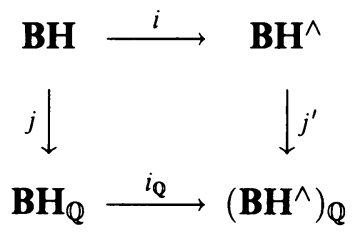

(see [BK,VI, 8.1]), where $\mathbf{X}^{\wedge}=\prod \mathbf{X}_{p}^{\wedge}$ is the product over all primes $p$ of the $p$-completions and $\mathbf{X}_{\mathbb{Q}}$ is the $\mathbb{Q}$-localization.

Without loss of generality, $i_{\mathbb{Q}}$ is a fibration and (3) is a pullback diagram, so both horizontal maps have the same fiber $\mathbf{F}$. Since $\mathbf{H}$ is compact and $\mathbf{B H}_{\mathbb{Q}}$, $\left(\mathbf{B H}^{\wedge}\right)_{\mathbb{Q}}$ are rational $H$-spaces, they are even-dimensional rational GEMs (that is, products of even-dimensional rational Eilenberg-Mac Lane spaces) and $\mathbf{F}$ is an odd-dimensional rational GEM.

For any map $f: \mathbf{B G} \rightarrow \mathbf{B H}$ (where $\mathbf{G}$ is a compact Lie group), (3) induces another pullback diagram

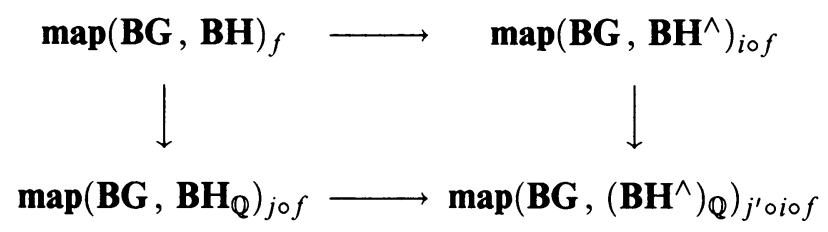

As for any compact Lie group, $H^{2 k-1}(\mathbf{B G} ; \mathbb{Q})=0$ for all $k \geq 1$ (cf. [Bo, Theorem 19.1]). Since $\mathbf{F} \simeq \prod \mathbf{K}\left(\mathbb{Q}, 2 r_{i}-1\right)$ is an odd-dimensional rational GEM, $\operatorname{map}(\mathbf{B G}, \mathbf{F})$ is an odd-dimensional rational GEM, too, by a direct calculation of its homotopy groups. In particular, $\operatorname{map}(\mathbf{B G}, \mathbf{F})$ is connected, and $\mathbb{F}_{p}$-acyclic for any prime $p$.

Thus $\operatorname{map}(\mathbf{B G}, \mathbf{F})$ is the fiber of $\operatorname{map}\left(\mathbf{B G}, \mathbf{B H}_{\mathbb{Q}}\right)_{c} \rightarrow \operatorname{map}\left(\mathbf{B G},\left(\mathbf{B} \mathbf{H}^{\wedge}\right)_{\mathbb{Q}}\right)_{c}$, where $c$ is the constant map. Because $\mathbf{B H}_{\mathbb{Q}}$ is an $H$-space and $i_{\mathbb{Q}}$ is an $H$ map, this is in fact the fiber for all components and thus for the two horizontal maps in (4).

Therefore, applying the $q$-completion functor to the top fibration sequence in the diagram

$$
\operatorname{map}(\mathbf{B G}, \mathbf{F}) \rightarrow \operatorname{map}(\mathbf{B G}, \mathbf{B H})_{f} \rightarrow \operatorname{map}\left(\mathbf{B G}, \mathbf{B H}^{\wedge}\right)_{i \circ f},
$$

we get another fibration (by [BK, II, 5.2]):

$$
\operatorname{map}(\mathbf{B G}, \mathbf{F})_{q}^{\wedge} \rightarrow\left(\operatorname{map}(\mathbf{B G}, \mathbf{B H})_{f}\right)_{q}^{\wedge} \stackrel{g}{\rightarrow}\left(\operatorname{map}\left(\mathbf{B G}, \mathbf{B H}^{\wedge}\right)_{i \circ f}\right)_{q}^{\wedge},
$$

with $g$ a homotopy equivalence (since the fiber is contractible).

Finally, Lemma 4.1 implies that $\left(\operatorname{map}\left(\mathbf{B G}, \mathbf{B H}_{p}^{\wedge}\right)_{i \circ f}\right)_{q}^{\wedge}$ is homotopy equivalent to $\left(\operatorname{map}\left(\mathbf{B G}, \mathbf{B H}_{p}^{\wedge}\right)_{i \circ f}\right)$ for $q=p$, and is contractible for $q \neq p$, so we get the desired homotopy equivalence

$$
\left(\operatorname{map}(\mathbf{B G}, \mathbf{B H})_{f}\right)_{p}^{\wedge} \cong \operatorname{map}\left(\mathbf{B G}, \mathbf{B H}_{p}^{\wedge}\right)_{i \circ f} .
$$

This completes the proof of Theorem 1.1. 


\section{REFERENCES}

[Bo] A. Borel, Sur la cohomologie des espaces fibrés principaux et des espaces homogènes de groupes de Lie compacts, Ann. of Math. (2) 57 (1953), 115-207.

[B1] A. K. Bousfield, The localization of spaces with respect to homology, Topology 14 (1975), 133-150.

[B2] — Homotopy spectral sequences and obstructions, Israel J. Math. 66 (1989), 54-104.

[BK] A. K. Bousfield and D. M. Kan, Homotopy limits, completions, and localizations, Lecture Notes in Math., vol. 304, Springer-Verlag, Berlin and New York, 1972.

[CE] H. Cartan and S. Eilenberg, Homological algebra, Princeton Univ. Press, Princeton, NJ, 1956.

[DZ] W. Dwyer and A. Zabrodski, Maps between classifying spaces, Algebraic Topology (Barcelona 1986), Lecture Notes in Math., vol. 1298, Springer-Verlag, Berlin and New York, 1989, pp. 106-119.

[Ha] D. K. Harrison, Infinite abelian groups and homological methods, Ann. of Math. (2) 69 (1959), 366-391.

[HS] P. J. Hilton and U. Stammbach, A course in homological algebra, Springer-Verlag, Berlin and New York, 1971.

[I] S. Illman, Equivariant singular homology and cohomology. I, Mem. Amer. Math. Soc. 156 (1975).

[JMO] S. Jackowski, J. E. McClure, and R. Oliver, Homotopy classification of self-maps of BG via G-actions, Aarhus Universitet Preprint Series, vol. 25, 1990.

[N1] D. Notbohm, Maps between classifying spaces, Math Z. 207 (1991), 229-257.

[N2] - Maps between classifying spaces and applications, Math. Gott. Heft 20 (1991).

[NS] D. Notbohm and L. Smith, Fake Lie groups and maximal tori. I, II, Math. Ann. 288 (1990), 637-661, 663-673.

Department of Mathematics, Northwestern University, Evanston, Illinois 60208

Current address, D. Blanc: Department of Mathematics, The Hebrew University, 91904 Jerusalem, Israel

Current address, D. Notbohm: SFB 170, Mathematisches Institut, 3400 Göttingen, Germany 